\title{
Correlation between bone and glucide metabolism depending on the nutritional status in diabetic patients
}

\author{
Alfieri Simone, $M S^{a, b}$ \\ a Department of clinical nutrition, Biomedical \\ Campus, Rome, Italy \\ ${ }^{b}$ Department of diabetology, Gemelli Hospital, \\ Rome, Italy \\ Received: $3^{\text {th }}$ October 2016.
}

\begin{abstract}
Introduction: The risk of osteoporotic fractures is increased in patients with either type 1 diabetes mellitus (T1DM) or type 2 diabetes mellitus (T2DM)

Aim: Evaluate the relationship between nutritional status, dairy calcium intake, serum levels of vitamin $D$, glycemic control, the onset of osteoporosis and/or bone fractures predisposition in diabetic patients.

Methods: The sample consists of 1151 patients (350 men and 801 women) divided into three groups: 400 patients with T1DM of which 19

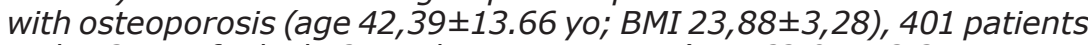
with T2DM of which 64 with osteoporosis (age 62.01土13.21 yo; BMI $30,25 \pm 8,83$ ), 350 non-diabetic patients with osteoporosis (NDP) (age $64.59 \pm 10.45$ yo; BMI 25,64 44,17$)$. In all subjects nutritional status, anthropometric, metabolic and glycemic control parameters, BMD (as $T$ - score) at the lumbar spine (LSBMD), femoral neck (FN-BMD) and total femur (Ftot-BMD) were measured. Prevalence of bone fractures between the different groups were determined.
\end{abstract}

Results and Discussion: Low vitamin D levels were found in both T1DM (16.38 $\pm 2.74 \mathrm{ng} / \mathrm{mL})$ and T2DM $(15.04 \pm 9.35 \mathrm{ng} / \mathrm{mL})$ as well as low daily calcium intake $(634.84 \pm 159.97 \mathrm{mg} /$ day and $649.43 \pm 189.86 \mathrm{mg} /$ day, respectively). About 89\% of T1DM and 37,5\% of T2DM had T-score $\geq-2,5$; T1DM had also a FN-BMD (Tscore: $-2.373 \pm 0.68$ vs $-1.91 \pm 0.72$; $p=0.016$ ) and Ftot-BMD (T-score: $-2.368 \pm 0.79$ vs $-1.60 \pm 0.96$; $p=0.003)$ significantly lower than T2DM and a LS-BMD significantly lower compared to NDP (T- score: $-2.26 \pm 0.79$ vs -2.91 \pm 0.86 ). Instead, T2DM had a LS-BMD, FN-BMD and Ftot-BMD significantly higher than those of NDP $(p=0.0001, p=0.004, p=0.007)$. We didn't found a positive correlation between BMD and HBA1c. 1\% of TIDM, 3.2\% of T2DM and $14.8 \%$ of NDP had vertebral fractures; $22.7 \%, 9.2 \%$ and $14 \%$ had nonvertebral fractures. T2DM had $38 \%$ reduction in risk of non-vertebral fractures (OR 0.62, 95\% CI=0.39- 0.98) compared with controls; instead, T1DM had an increased risk of non-vertebral fractures (OR 1.81, 95\% CI 1.24-2.66).

Conclusions: We confirm that T1D had an increased risk of fractures. Calcium intake and vitamin $D$ resulted insufficient in all groups. HBA1c did not affect BMD or risk of fractures in all groups.

Keywords: Diabetes, Osteoporosis, Fracture, Nutritional Status

\section{INTRODUCTION}

Since Albright first proposed the concept of "diabetic osteopenia", many studies have investigated the levels of bone mineral density (BMD) and the risk of osteoporosis in type 1 and type 2 diabetes [1]. Diabetes mellitus and low-trauma fractures are the major causes of morbidity and premature mortality worldwide. Although several observational studies have been investigated the association between diabetes and risk of fractures, the role of diabetes as a potential risk factor for osteoporosis and low- trauma fractures remains unsettled [1].

The risk of osteoporotic fractures is increased in patients with both type 1 diabetes mellitus (T1DM) and type 2 diabetes mellitus (T2DM). Although BMD is decreased in T1DM, BMD in T2DM is often normal or even slightly elevated compared to an age-matched control population. However, in both
T1DM and T2DM, bone turnover is decreased and the bone material properties and microstructure of bone are altered. This is reflect- ed in a decrease in serum markers of bone formation, such as osteocalcin [2]. However, the pathophysiological mechanisms underlying bone fragility in diabetes mellitus are complex, and include hyperglycemia, oxidative stress and the accumulation of advanced glycation end products that compromise collagen properties, increase marrow adiposity, release inflammatory factors and adipokines from visceral fat, and potentially alter the function of osteocytes. Additional factors including treatment-induced hypoglycemia, different antidiabetic medications with a direct effect on bone and mineral metabolism (such as thiazolidinediones), as well as an increased predisposition to falls, all contribute to the increased fracture risk in patients with diabetes [2].

In particular, T1DM does appear to be a significant risk factor for osteoporosis (OP). Many 
of the nutrients and food components we consume as part of a Westernized diet can potentially have a positive or negative impact on bone health. They may influence bone by various mechanisms, including alteration of bone structure, the rate of bone metabolism, the endocrine and/or paracrine system, and homeostasis of calcium and possibly of other bone-active mineral elements. These dietary factors range from inorganic minerals (e.g., calcium, magnesium, phosphorus, sodium, potassium, and various trace elements) and vitamins (vitamins A, D, E, K, C, and certain B vitamins), to macronutrients, such as protein and fatty acids. Protein has been identified as being both detrimental and beneficial to bone health, depending on a variety of factors, including the level of proteins in the diet, the protein source, calcium intake, weight loss, and the acid/ base balance of the diet. Currently, we recommend that patients with both types of diabetes must be monitored more carefully than persons without diabetes, and should be encouraged to consume a diet rich in both calcium (at least $1200 \mathrm{mg} /$ day) and vitamin D (400-600 IU/day). Adequate calcium intake is critical to achieving optimal peak bone mass and modifies the rate of bone loss associated with aging. There is a considerable body of evidence that vitamin $\mathrm{D}$ deficiency is an important contributor to osteoporosis through less efficient intestinal absorption of calcium, increased bone loss, muscle weakness, and a weakened bone microstructure. Increasing vitamin $D$ intake can significantly reduce risk of bone fracture in older people.

It appears that intensive insulin therapy and a stable body weight in patients with type 1 diabetes is important to prevent bone loss [3].

The aim of this study was to evaluate the relationship between nutritional status, dairy calcium intake, serum levels of vitamin D, glycemic control, the onset of osteoporosis and/or bone fractures predisposition in diabetic patients. In addition, the differences between diabetic and non diabetic patients regards to anthropometric and physiologic parameters in relation to the onset of fractures were evaluated.

\section{MATERIAL AND METHODS}

Between November 2014 and July 2015, a total of 1151 patients were enrolled. According to the National Health and Nutrition Examination Survey, in all subjects, anthropometric parameters (age, BMI, waist and wrist circumference), cardio-metabolic parameters (basal metabolism, diastolic and systolic pressure, total cholesterol, HDL, LDL, triglycerides, serum levels of vitamin D) and parameters related to glycemic control (fasting glucose and HbA1c) were first evaluated. Then, parameters related to nutritional status, like energy intake and nutrients intake, were determined throughout a validated food frequency questionnaire.

BMD (as T-score) at the lumbar spine (LS$B M D$ ), at femoral neck (FN-BMD) and at total femur (Ftot- BMD) were also measured with Dual energy $X$ Ray Absorptiometry. The risk factors involved in the etiology of osteoporosis and the prevalence of bone fracture between the different groups were deter- mined throughout two different quality of life questionnaires: the QUALEFFO-41 was used for patients with vertebral fractures [4], and the IOF Wrist Fracture Questionnaire was used for patients with wrist fractures [5]. For what concerning statistical analyses, the characteristics of all participants were compared to identify the main differences related to the anthropometric and physiological parameters between the different groups surveyed. Subsequently, the characteristics of type 1 diabetes mellitus (TIDM) and type 2 diabetes mellitus (TIIDM) patients were compared to identify the differences between the two groups and, after this, they wer e compared with non-diabetic patients (NDP). The IBM SPSS statistical software has been used, using model Analysis of Variance (ANOVA), which allows the analysis of variance of the various parameters in relation to the observed variables. In the case in which the variable considered is linked in a statistically significant manner to the variable of interest, this will be expressed by a p-value (probability of obtaining a result equal to or more extreme than the one observed, suppository true the null hypothesis, that is, the hypothesis you want to check in the test) equal to or less than 0.05 .

\section{RESULTS}

In the period between November 2013 and July 2014, a total of 1151 patients were enrolled in this study. Among them, 350 (30\%) were men and 801 (70\%) women. The patients were divided into three groups ac- cording to their pathology. The first group consists of 400 patients with T1DM of

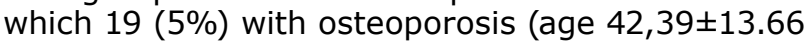
yo; BMI $23,88 \pm 3,28)$, the second group consists of 401 patients with T2DM of which $64(16 \%)$ with osteoporosis (age 62.01 \pm 13.21 yo; BMI $30,25 \pm 8,83$ ) and the third group consists of

350 non-diabetic patients with osteoporosis (NDP) (age $64.59 \pm 10.45$ years; BMI 25,64 $\pm 4,17$ ). It was observed that the age of patients with T1DM was significantly lower compared to both patients with T2DM ( $p=0.0001)$ and non-diabetic patients $(p=0.02)$. As expected, both BMI $(p=0.0001)$ and waist circumference $(p=0.0001)$ were higher in T2DM patients than the other two groups.

Regard to the cardio-metabolic parameters, patients with T2DM showed higher value of systolic blood pressure, total cholesterol (202.8 \pm 38.63 vs. $175.17 \pm 23,79 ; p=0.0001)$, LDL cholesterol $(151.69 \pm 37.05$ vs $129.26 \pm 24.54 ; \mathrm{p}=0.001)$ and triglycerides $(117.38 \pm 38.17$ vs $72.71 \pm 21,62 ; p$ $=0.0001)$, but lower level of $\operatorname{HDL}(27,63 \pm 31,37$ \pm 5.6 vs 7.5; $p=0.0001$ ) than patients with T1DM. The serum levels of vitamin D were higher in nondiabetic patients compared to both T1DM (21.18 \pm $9.75 \mathrm{vs} 16.38 \pm 2.74 \mathrm{ng} / \mathrm{mL}$ ) and T2DM patients $(21.18 \pm 9.75$ vs. $15.04 \pm 9.35 \mathrm{ng} / \mathrm{mL})$. However, all patients showed an inverse correlation between serum levels of vitamin D and their age. All groups presented low level of this vitamin (vitamin $D<30$ $\mathrm{ng} / \mathrm{ml}$ ).

Regard to the nutritional status, the comparison between T1DM patients and T2DM patients showed a significant difference between all parameters, except for daily calcium intake. For what concerning this parameter, examination of the data showed that diabetic subjects had higher daily calcium intake than non-diabetics (DMI vs OP: $634.84 \pm 159.97$ 
$\mathrm{mg} /$ day vs $592.21 \pm 130.72 \mathrm{mg} /$ day ; $\mathrm{p}=0.0001)$ (DMII vs OP: $649.43 \pm 189.86 \mathrm{mg} /$ day vs 592.21 $\pm 130.72 \mathrm{mg} /$ day; $\mathrm{p}=0.003$ ) (Fig. 1). Further, a significant correlation between daily calcium intake, BMI $(p=0.001)$ and waist circumference $(p=0.011)$ was observed in patients with T1DM. About daily caloric intake, as expected, it was higher in patients with T2DM, who consume a lot of animal protein $(p=0.0001)$, than other groups.

Regards to the analysis of the bone mineral density, patients with T1DM had a femoral neck (T-score: $-2.373 \pm 0.68$ vs $-1.91 \pm 0.72 ; p=0.016$ ) and total hip BMD (T-score: $-2.368 \pm 0.79$ vs -1.60 $\pm 0.96 ; p=0.003$ ) lower than patients with T2DM, and they have lower column BMD compared to nondiabetic patients (T- score: $-2.26 \pm 0.79$ vs. -2.91 $\pm 0.86)$. Instead patients with T2DM had lumbar spine, femoral neck and total hip BMD higher than non-diabetic with osteoporosis patients $(p=$ $0.0001, p=0.004 ; p=0.007$ ). Patients with T1DM showed different cardio-metabolic parameters and a significant correlation between BMD ana- lyzed in various locations and the basal metabolism of the diabetic. In the T2DM patients a correlation between BMD at lumbar spine and basal metabolism with vitamin D was observed. Instead in nondiabetic group a significant correlation between femoral neck BMD and vitamin D level emerged. Further analyzing the values related to the glycemic control (fasting blood glucose T2DM: $182.67 \pm 74.59$ vs T1DM: $136.64 \pm 56.60$ vs OP: $92.29 \pm 20.87 \mathrm{mg}$ $/ \mathrm{dL}$ ) (glycated hemoglobin T2DM : $8.12 \pm 2.31 \mathrm{vs}$ T1DM: $7.15 \pm 0.77 ; p=0.0001$ ) in both diabetes patient groups, it seems that these parameters did not affect BMD. In addiction, no significant correlations emerged between BMD and the factors related to the glycemic control (fasting blood glucose and glycated hemoglobin). It seems that there was no significant correlation between BMD and parameters related to the nutritional status in patients with T1DM, whilst BMD of the total femur is significantly correlated to the percentage of animal protein and sodium intake in patients with T2DM. Furthermore, no significant correlation between BMD and daily calcium intake emerged.

Regard to the fractures, 93 patients with only osteoporosis $(26,60 \%) ; 19$ patients with both T1DM

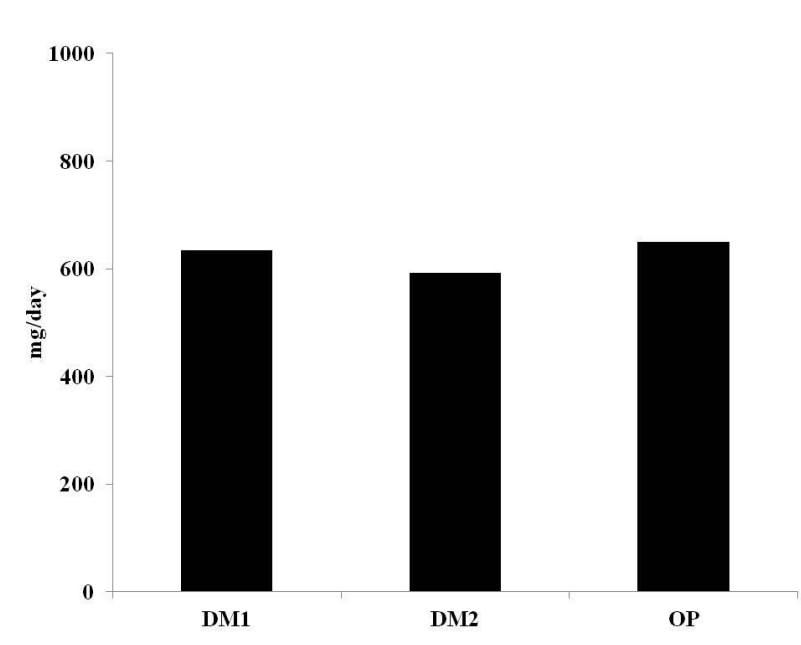

Fig. 1 Daily calcium intake in all groups $\mathrm{DM} 1=$ type 1 diabetes $\mathrm{DM} 2$ = type 2 diabetes $\mathrm{OP}=$ osteoporosis and osteoporosis (4.75\%) and 44 patients with T2DM and osteoporosis $(11 \%)$ had at least one fracture (Fig. 2). Fractures analysis showed that respectively $1 \%$ T1DM patients, $3.2 \%$ of T2DM and $14.8 \%$ of NDP had vertebral fractures; $22.7 \%, 9.2 \%$ and $14 \%$ had non-vertebral fractures and $23.2 \%$, $10.2 \%$ and $26.9 \%$ had total fractures in the three different groups. Furthermore, a logistic regression for different fractures was performed as reported in table 1 . Patients with T1DM showed an increased risk of fractures with an OR of 1.81 (95\% CI 1.24 to 2.66) compared to controls. Instead, patients with T2DM showed an odds ratio (OR) of 0.62 ( $95 \%$ confidence interval $(\mathrm{CI})=0.39$ to 0.98 ) and a reduced risk of $38 \%$ related to the non-vertebral fractures compared to controls.

\section{DISCUSSION}

Several studies have shown that there is a correlation between bone and glucose metabolism in patients with diabetes who may reflect an increased risk of fractures, nevertheless the mechanisms involved are not yet fully known. Diabetes can affect bone metabolism via different mechanisms, many of which can have contradictory effects. In this context, the aim of this study was to evaluate the relationship between nutritional status, dairy calcium intake, serum levels of vitamin D, glycemic control, the onset of osteoporosis and/or bone fractures predisposition in diabetic patients. In addition, the differences between diabetic and non diabetic patients regards to anthropometric and physiologic parameters in relation to the onset of fractures wer e evaluated.

The serum levels of vitamin D observed were significantly higher in non-diabetic patients compared to patients with both types of diabetes. However, all groups of patients had low levels of vitamin $\mathrm{D}$ (vitamin $\mathrm{D}<30 \mathrm{NG} / \mathrm{ml}$ ). In addition, both groups of diabetic patients analyzed had lower values of vitamin $D$ compared to patients with osteoporosis and it seems to be inversely correlated to age. To reduce the risk of fractures in diabetic patients is necessary to ensure an adequate intake of vitamin $D$; its deficit can increase the risk of

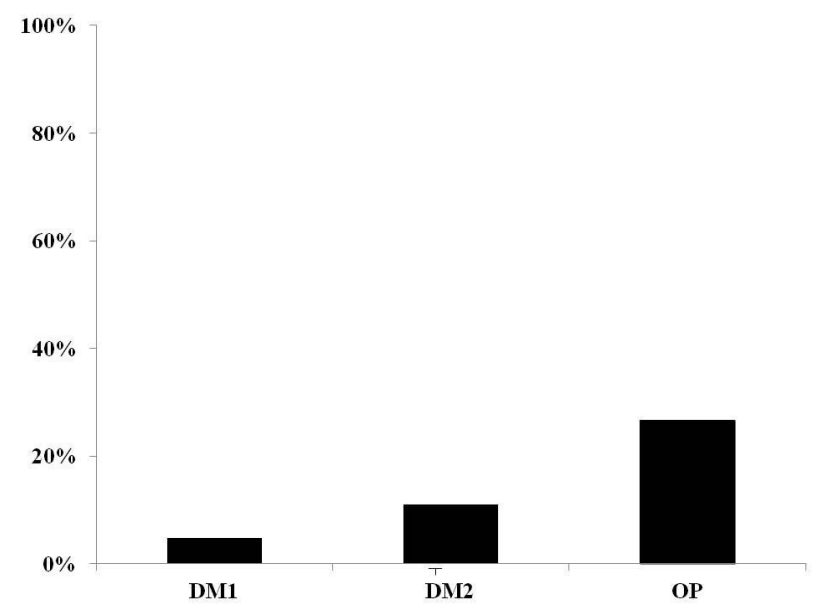

Fig. 2 Percentage of patients with almost one fracture in the different groups

DM1 = type 1 diabetes DM2= type 2 diabetes $\mathrm{OP}=$ osteoporosis 
fractures. The recommended quantity of vitamin $\mathrm{D}$ in adults and elderly should be about $30 \mu \mathrm{g} / \mathrm{day}$, since it reduces the risk of falls and fractures by 20 and $23 \%$. The main source of vitamin D for humans is the light exposure throughout endogenous synthesis. The mechanisms involved in vitamin $D$ deficiency in obese subjects are not fully known; it has been hypothesized that the reduced exposure to sunlight and low intake in the diet can contribute to this deficiency. Moreover, it is likely that the excessive surface fat present in the obese, act as a depot, limiting the circulating levels of vitamin $D$. In addition, a deficiency of vitamin $D$ could play a role in the development of insulin resistance and, consequently, in type II diabetes mellitus. Some studies report that patients with low levels of vitamin $D$ have lower insulin sensitivity than those with normal levels. Although the role of vitamin $D$ in the onset of diabetes mellitus is not entirely known, several studies have shown that an adequate daily intake of vitamin $D$ could reduce the risk of developing diabetes through stimulation of insulin synthesis at the level of beta cells of the pancreas, stimulating the expression of insulin receptors or regulating calcium homeostasis, which is essential for intracellular signals mediated by insulin.

Furthermore, calcium intake was significantly higher in patients with diabetes compared to nondiabetic and was positively correlated to BMI, glycated hemoglobin and waist circumference. The adequate calcium in- take is fundamental to reduce the risk of osteoporosis and the onset of the fractures in patients with diabetes. The daily recommended levels of calcium intake are $1000 \mathrm{mg} /$ day for healthy adults and $1200 \mathrm{mg} /$ day for elderly people, because, with age, the absorption of calcium in the intestine decreases.

The most important factor related to the calcium intake regards its bioavailability, the fraction of dietary calcium that is potentially absorbable and the incorporation of the absorbed calcium into bone.

Various dietary factors can affect calcium bioavailability. Some food components act synergistically to pro- mote calcium absorption. They include: vitamin $D$, lactose and casein phosphopeptides present in milk [6]. Instead, other food components, such as phytates, oxalates, polyphenols (such as the tannic and caffeic acid), fatty acids and dietary fiber, may limit the bioavailability of this microelement. For this reason, some plant foods, although they are good sources of calcium, in terms of absolute content, they do not provide coverage of the recommended requirements.

Regards to the BMD observed, the results of this study confirmed that patients with diabetes type I had low levels of BMD at all sites compared to diabetes type II patients. The risk of fractures in type I diabetes is due primarily, to a reduction in BMD that results from an absolute deficiency of insulin and IGF-1 and which results in low values of peak bone mass. In patients with T2DM, obesity and insulin resistance leads to in- creased bone formation. Regards to the nutritional status, the BMD of the total femur of patients with T2DM appears to be related to both the percentage of animal protein and the sodium intake; whilst in no group ther e was a correlation between BMD and daily calcium intake. Nevertheless, patients with T2DM showed a BMD of the spine, total femur and femoral neck higher than non-diabetic patients.

In addition, an inadequate glycemic control for both groups of diabetic patients, despite their dietary and drug treatment, was observed but it did not reveal any correlation between BMD and the fundamental factors related to glycemic control (fasting blood glucose and glycated hemoglobin).

Finally, the greater bone loss observed in diabetic patients leads to an increased risk of spontaneous pathological fractures. In the present study, the prevalence of fractures appears to be greater in nondiabetic patients compared to those with diabetes, probably due to the small number of the sample. Moreover, patients with T2DM had a reduced risk of $38 \%$ compared to patients with T1DM regards to the non-vertebral fractures. Starting from these results, it is reasonable to carry out a broader screening for diabetes patients in the future. The ineffectiveness of BMD in assessing fractures risk in patients with T2DM leads to problems to perform risk assessment and to initiate therapy for the prevention of fractures in clinical practice. Although there are several candidates (osteocalcin, AGEs and insulin) for this purpose, yet it is unclear whether they can effectively be used in clinical practice.

\section{CONCLUSIONS}

The lack of effective treatments for degenerative diseases such as osteoporosis places increased emphasis on a preventative approach, including

Table 1. Analysis offractme risk in patients with T1DM and T2DM compared with controls

\begin{tabular}{ccccc}
\hline & frattw. & OR & IC \\
\hline \multirow{2}{*}{ T1DM } & FX non vert. & 1,80 & 1,24 & 2,67 \\
& Fx vert. & 0,058 & 0,02 & 0,14 \\
\cline { 2 - 5 } & Fx tot. & 0,83 & 0,59 & 1,15 \\
\hline \multirow{2}{*}{ T2DM } & FX non vert. & 0,62 & 0,39 & 0,98 \\
& Fx vert. & 0,19 & 0,99 & 0,35 \\
\hline & Fx tot. & 0,31 & 0,21 & 0,46 \\
\hline
\end{tabular}

$\mathrm{OR}=$ odds ratio, $\mathrm{IC}=$ confidence interval 
dietary strategies. The therapeutic considerations in diabetic patients with osteoporosis may be considered simple recommendation of "good clinical practice" evidence based and they should include: maintaining a good control of glycaemia, minimizing hypoglycemic episode, preventing diabetes complication, especially renal pathology, evaluating and prevent falls, supplementing calcium and vitamin $D$, practicing physical activity to minimize the risk of falls, using anti-osteoporotic treatment like alendronate.

In conclusion, to achieve a good glycemic control throughout proper nutrition and an adequate level of physical activity in patients with diabetes appears to be the best way to prevent and treat this disease.

\section{ACKNOWLEDGEMENTS}

The author gratefully acknowledge the diabetology department of Gemelli, Rome, Italy and the endocrinology department of Biomedical Campus, Rome, Italy.

\section{Conflicts of interest}

The author declare that they have no competing interests.

\section{REFERENCES}

1. Janghorbani et al, Systematic Review of Type 1 and Type 2 Diabetes Mellitus and Risk of Fracture Am. J. Epidemiol. (2007) 166 (5): 495505

2. Napoli N et al., Mechanisms of diabetes mellitusinduced bone fragility. Nat Rev Endocrinol. 2016 Sep 23.

3. Campos Pastor et al., Intensive insulin therapy and bone mineral density in type 1 diabetes mellitus: a prospective study. Osteoporos Int. 2000;11(5):455-9.

4. Tadic I et al., Validation of the osteoporosis quality of life questionnaire QUALEFFO-41 for the Serbian population. Health Qual Life Outcomes. 2012 Jun 18;10:74

5. Lips $\mathrm{P}$ et al., Validation of the IOF quality of life questionnaire for patients with wrist fracture. Osteoporosis Int. 2010 Jan;21(1):6170

6. Caroli A et al. Invited review: Dairy intake and bone health: A viewpoint from the state of the art. J Dair Sci2011;94(11): 